Homology, Homotopy and Applications, vol.11(1), 2009, pp.115-140

\title{
LOCAL ACYCLIC FIBRATIONS AND THE DE RHAM COMPLEX
}

\author{
BEN LEE
}

(communicated by Michael A. Mandell)

Abstract

We reinterpret algebraic de Rham cohomology for a possibly singular complex variety $X$ as sheaf cohomology in the site of smooth schemes over $X$ with Voevodsky's $h$-topology. Our results extend to the algebraic de Rham complex as well. Our main technique is to extend Čech cohomology of hypercovers to arbitrary local acyclic fibrations of simplicial presheaves.

\section{Introduction}

Let $X$ be a separated scheme finite type over the complex numbers $\mathbb{C}$. Following Deligne, Du Bois ([5]) constructs the algebraic de Rham complex of $X$

$$
\Omega_{X / \mathbb{C}}^{\bullet}:=R e_{*} \Omega_{X \cdot / \mathbb{C}}^{\bullet}
$$

by a choice of a smooth proper hypercover. It is well-defined in the filtered derived category. Morally, this Cech complex should be a derived direct image from some topos to the Zariski site; showing this is the aim of this paper.

The choice of topos appears to be a delicate matter. Using the topology of "universal cohomological descent" (which we abbreviate "ucd") on proper and smooth schemes turns out to be technically inconvenient. We use instead Voevodsky's $h$-topology [22] on possibly open schemes. Denote by $\mathrm{Sm}_{h} / X$ the category of smooth separated schemes finite type over $X$, equipped with the $h$-topology. We show that the presheaf $\Omega^{q}$ is a sheaf on $\operatorname{Sm}_{h} / X$. There is a direct image $\gamma_{*}$ from sheaves on $\mathrm{Sm}_{h} / X$ to sheaves on the small Zariski site $X_{\text {Zar }}$.

Unfortunately, we cannot directly apply Verdier's work on Čech cohomology of hypercovers. Comparing Čech and derived functor cohomology in this situation requires finite fiber products which do not exist in Sm. However, the standard comparison would show that

$$
\underline{\Omega_{X / \mathbb{C}}^{\bullet}} \simeq R \gamma_{*} \Omega^{\bullet}
$$

and thus

$$
H_{d R}^{i}(X) \simeq \mathbf{H}_{h}^{i}\left(X, \Omega^{\bullet}\right)=\mathbf{H}_{\mathrm{Zar}}^{i}\left(X, R \gamma_{*} \Omega^{\bullet}\right)
$$

giving our main result. (By GAGA [10] and the results of [9], this would be isomorphic to its analytic counterpart.)

Received March 3, 2008, revised October 17, 2008; published on May 4, 2009.

2000 Mathematics Subject Classification: 14F40, 55N30.

Key words and phrases: simplicial presheaves, de Rham cohomology, homotopy.

This article is available at http://intlpress.com/HHA/v11/n1/a6

Copyright (C) 2009, International Press. Permission to copy for private use granted. 
According to Jardine ([18]), hypercovers are just (semi-)representable local acyclic fibrations. Keeping this in mind, we generalize Verdier's work on Čech cohomology to arbitrary local acyclic fibrations of simplicial presheaves. The precise statement proved in the first section is

Theorem 1.1. Let $X_{\bullet}$ be a simplicial presheaf, and $h D\left(X_{\bullet}\right)$ the homotopy category of local acyclic fibrations $K_{\bullet} \rightarrow X_{\bullet}$. Then for a bounded below complex of sheaves of abelian groups $\mathscr{F} \bullet$ with the filtration bete there is an isomorphism

$$
\underset{\lim _{\bullet} \in \overrightarrow{h D}\left(X_{\bullet}\right)}{ } H^{p}\left(\operatorname{Tot} \operatorname{Hom}\left(\mathbb{Z} K^{\bullet}, \mathscr{F} \bullet\right)\right) \operatorname{Ext}^{p}\left(\mathbb{Z} X^{\bullet}, \mathscr{F} \bullet\right)
$$

and there is a filtered quasi-isomorphism of ind-objects in the derived category

$$
\operatorname{ind}_{K \bullet \in h D\left(X_{\bullet}\right)} \operatorname{Tot} \operatorname{Hom}\left(\mathbb{Z} K^{\bullet}, \mathscr{F} \bullet\right) \simeq \operatorname{Hom}\left(\mathbb{Z} X^{\bullet}, \mathscr{F} \bullet\right) .
$$

Note the lack of hypotheses on fiber products in the underlying topos.

In practice, one usually wants to restrict to local acyclic fibrations which satisfy some representability hypothesis. Define a semi-representable presheaf to be a presheaf that is isomorphic to a coproduct of representable presheaves; that one can restrict to semi-representable presheaves is an easy corollary of the above theorem. To satisfy stronger hypotheses than semi-representability seems to require something from the underlying topos - in our case we use the inclusion $\mathrm{Sm}_{h} \subset \mathrm{Sch}_{h}$, and resolution of singularities or alterations $([2,17])$.

Section 3 is occupied with "topological" matters. Using Du Bois' results requires a comparison of the $u c d$ - and $h$-topologies: after some preliminaries, we show every $h$-covering is a $u c d$-covering. We do not know of an example of a $u c d$-covering that is not an $h$-covering. Finally, we show one can actually compute using representable presheaves in $\mathrm{Sm}_{h}$.

In Section 4 we apply our work to the algebraic de Rham complex. Key in applying Du Bois' results is Theorem 4.13, which compares $h$-hypercovers to Zariski hypercovers. This result comes from a generous suggestion of Alexander Beilinson. Also in this section is the proof that $\Omega^{q}$ is a sheaf in the $h$-topology. These results with the Čech theory yield the main theorem.

We end with some questions which spurred our investigations, and potentially point to new directions. Eventually we hope to find connections with Hodge theory for open varieties, intersection cohomology, and further examples of the applications of homotopical methods to algebraic geometry.

\section{Acknowledgements}

This paper is based on my dissertation, and I owe thanks to the many people who helped me. Everything here has benefited from the guiding hand of my advisor, Madhav Nori, to whom I give my sincerest thanks. Alexander Beilinson has also provided invaluable help and advice. I would also like to thank Andrew Blumberg and Minhea Popa for stimulating mathematical discussions, and the reviewer for his careful reading and useful suggestions. 


\section{A generalized Verdier theorem}

\subsection{Local acyclic fibrations}

Let $C$ be a site, Pre $C$ the category of presheaves of sets on $C$, Sh $C$ the category of sheaves of sets on $C$, and $s \operatorname{Pre} C, s \operatorname{Sh} C$ the categories of simplicial presheaves and sheaves. Note that, unlike [12, ex V 7.3.0], we do not assume the existence of products and finite fiber products in our site $C$. Let $e$ be the terminal object of $\operatorname{Sh} C$. For a presheaf $K$, let $\mathbb{Z} K$ denote the associated sheaf of free abelian groups; for a simplicial presheaf $K_{\bullet}$, let $\mathbb{Z} K^{\bullet}$ denote the associate negative cochain complex of sheaves of free abelian groups; define $\mathbb{Z}:=\mathbb{Z} e$ as the sheaf of free abelian groups associated to the terminal object $e$.

Definition 2.1 (cf. $[6,7,18]$ ).

1. Let $f: L_{\bullet} \rightarrow K_{\bullet}$ be a morphism of simplicial presheaves. $f$ is called a local acyclic fibration if, for every $U \in C$, integer $k \geqslant 0$ and diagram

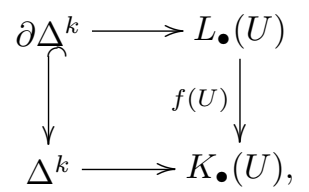

there is a refinement (a covering sieve) $R$ of $U$ so that for every $V \rightarrow U \in R$ there is a lift

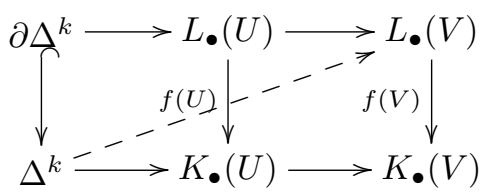

indicated by the dashed arrow. We say $f$ satisfies the local right lifting property for the inclusion $\partial \Delta^{k} \rightarrow \Delta^{k}$.

2. For a presheaf $M \in \operatorname{Pre} C$, let $M_{\bullet}$ be the simplicial presheaf which is $M$ in all degrees. We abuse notation and call the augmented simplicial presheaf $K_{\bullet} \rightarrow M$ a local acyclic fibration if the morphism of simplicial presheaves $K_{\bullet} \rightarrow M_{\bullet}$ is a local acyclic fibration.

3. Recall that a simplicial presheaf is semi-representable if its components are isomorphic to coproducts of representable presheaves. A local acyclic fibration $L_{\bullet} \rightarrow K_{\bullet}$ is a hypercover if both $L_{\bullet}$ and $K_{\bullet}$ are semi-representable.

Compare the following with [12, ex V Lemma 7.3.6]:

Lemma 2.2. A morphism $f: L_{\bullet} \rightarrow K_{\bullet}$ is a local acyclic fibration if and only if for every $P_{\bullet} \hookrightarrow Q_{\bullet}$ an inclusion of constant simplicial sets with only finitely many nondegenerate simplices, we can locally lift diagrams

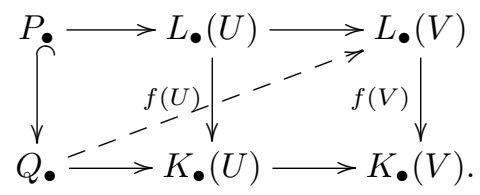


Proof. Induction on the definition.

Recall a morphism of presheaves $F \rightarrow G$ is a covering morphism if the associated morphism of sheaves is an epimorphism (see [11, II.5.2]).

Remark 2.3. For a morphism $f: L_{\bullet} \rightarrow K_{\bullet}$, Verdier uses the following equivalent definition of local acyclic fibration (which he calls "special"):

1. For each integer $k \geqslant 0$, the morphism $\phi_{k}$ in the diagram is a covering morphism:

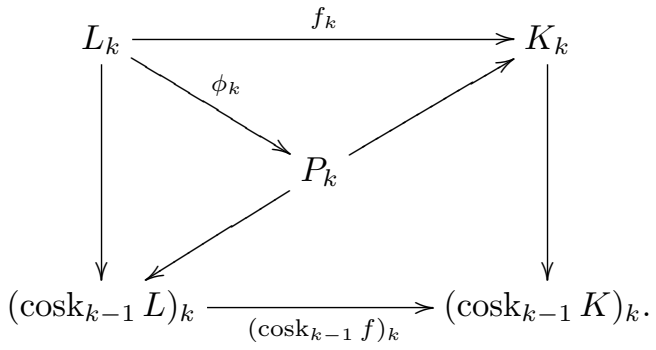

The vertical arrows are the coskeleton adjunction maps and $P_{k}$ is the fiber product of $K_{k}$ and $\left(\operatorname{cosk}_{k-1} L\right)_{k}$ by the arrows in the diagram.

2. The morphism $f_{0}: L_{0} \rightarrow K_{0}$ is a covering morphism.

For a simplicial set $S_{\bullet}$, let $\underline{S}_{\bullet}$ denote the simplicial presheaf whose value in degree $d$ is always $S_{d}$. Recall that $\mathscr{H} \operatorname{om}\left(L_{\bullet}, K_{\bullet}\right)(U)=\operatorname{Hom}\left(F_{\bullet}(U), G_{\bullet}(U)\right)$ is presheaf Hom, and $\operatorname{sHom}\left(L_{\bullet}, K_{\bullet}\right)_{n}=\mathscr{H} \operatorname{om}\left(L_{\bullet} \times \underline{\Delta}^{n}, K_{\bullet}\right)$ is the internal Hom in the category of simplicial presheaves.

Proposition 2.4 ([7, Proposition 7.2]). Let $f: L_{\bullet} \rightarrow K_{\bullet}$ be a morphism of simplicial presheaves. Then the following are equivalent:

1. $f$ is a local acyclic fibration.

2. For every integer $k \geqslant 0$, the morphism

$$
\mathscr{H} \mathrm{om}\left(\underline{\Delta}^{k}, L_{\bullet}\right) \rightarrow \mathscr{H} \mathrm{om}\left(\underline{\partial \Delta^{k}}, L_{\bullet}\right) \times \mathscr{H} \mathrm{om}\left(\underline{\partial \Delta^{k}}, K_{\bullet}\right) \mathscr{H} \mathrm{om}\left(\underline{\Delta}^{k}, K_{\bullet}\right)
$$

induced by the inclusion $\partial \Delta^{k} \rightarrow \Delta^{k}$ and $f$ is a covering morphism.

3. $f$ is special in the sense of Verdier.

Proof. $1 \Leftrightarrow 2$ is by definition. To show $2 \Leftrightarrow 3$, we apply the isomorphisms $X_{k}=$ $\operatorname{Hom}\left(\Delta^{k}, X_{\bullet}\right)$ and the coskeleton-skelton adjunction to the covering condition

$$
L_{k} \rightarrow\left(\operatorname{cosk}_{k-1} L\right)_{k} \times\left(\operatorname{cosk}_{k-1} K\right)_{k} K_{k}
$$

noting that $\operatorname{sk}_{k-1} \underline{\Delta}^{k}=\underline{\partial \Delta^{k}}$.

We recall the following basic results.

Proposition 2.5 ([18, Proposition 2.9]). If $f: L_{\bullet} \rightarrow K_{\bullet}$ is a local acyclic fibration of simplicial presheaves, then the induced map $\mathbb{Z} L^{\bullet} \rightarrow \mathbb{Z} K^{\bullet}$ is a quasi-isomorphism of complexes of sheaves. 
Lemma 2.6 ([12, ex V Lemma 7.3.4]).

1. The composition of two local acyclic fibrations is a local acyclic fibration.

2. Local acyclic fibrations are preserved under base change.

3. Suppose $K_{\bullet} \rightarrow X_{\bullet}$ is a local acyclic fibration, $f: L_{\bullet} \rightarrow M_{\bullet}$ a local acyclic fibration, and $K_{\bullet} \rightarrow M_{\bullet}$ a morphism. Then $L_{\bullet} \times_{M_{\bullet}} K_{\bullet} \rightarrow X_{\bullet}$ is a local acyclic fibration.

\subsection{Computing Ext}

Before proving our main theorem we require a technical lemma. First, some notation: Let $C$ be an abelian category, and $f: I \rightarrow D^{+}(C)$ be a filtered system in the derived category of $C$. The associated ind-object is denoted by

$$
\operatorname{ind}_{M \bullet \in f(I)} M^{\bullet} \text {. }
$$

We define the cohomology of this ind-object by the equation

$$
H^{k}\left(\operatorname{ind}_{M \bullet \in f(I)} M^{\bullet}\right):=\lim _{M \bullet \in f(I)} H^{k}\left(M^{\bullet}\right) .
$$

We note that, in the case where the ind-object is representable, this agrees with the cohomology of the colimit object since $H^{k}\left(\lim ^{\bullet} M^{\bullet}\right)=\lim _{\longrightarrow} H^{k}\left(M^{\bullet}\right)$, cf. [19, 1.12.7],

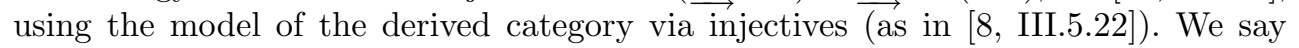
that a map of ind-objects is a quasi-isomorphism if it induces an isomorphism on cohomology.

The main results of this subsection are:

Lemma 2.7 (Lemma on computing Ext). Let $C$ be an abelian category with enough injectives, $X^{\bullet} \in \mathrm{Ch}^{-}(C)$ a fixed negative cochain complex, and $G^{\bullet} \in \mathrm{Ch}^{+}(C)$ a fixed positive cochain complex. Suppose $D \subset \mathrm{Ch}^{-}(C) / X^{\bullet}$ is a subcategory of the category of negative cochain complexes of $C$ over $X^{\bullet}$ with the following properties:

1. The homotopy category $h D$ (morphisms up to chain homotopy) is cofiltered.

2. For every complex $K^{\bullet} \in D$, object $M \in C$, and epimorphism $u: M \rightarrow K^{n}$, there is a complex $L^{\bullet} \in D$ and a morphism $f: L^{\bullet} \rightarrow K^{\bullet}$ whose degree $n$ part factors as

$$
f^{n}: L^{n} \rightarrow M \stackrel{u}{\rightarrow} K^{n}
$$

3. Every $K^{\bullet} \in D$ has structure morphism $K^{\bullet} \rightarrow X^{\bullet}$ a quasi-isomorphism.

Then the functor $\operatorname{ind}_{K^{\bullet} \in h D} \operatorname{Tot} \operatorname{Hom}_{C}\left(K^{\bullet}, \ldots\right)$ preserves quasi-isomorphisms and there is a filtered isomorphism of ind-objects in the derived category

$$
\operatorname{ind}_{K^{\bullet} \in h D} \operatorname{Tot} \operatorname{Hom}_{C}\left(K^{\bullet}, G^{\bullet}\right) \simeq R \operatorname{Hom}_{C}\left(X^{\bullet}, G^{\bullet}\right)
$$

(where on each side the filtration arises from the filtration bête on $G^{\bullet}$ ). This induces for each $p$ an isomorphism of functors

$$
\lim _{K^{\bullet} \in h D} H^{p}\left(\operatorname{Tot} \operatorname{Hom}_{C}\left(K^{\bullet}, G^{\bullet}\right)\right) \simeq \operatorname{Ext}_{C}^{p}\left(X^{\bullet}, G^{\bullet}\right) .
$$

Here Ext is hyper-Ext. 
Proof. Given an injective resolution $G^{\bullet} \rightarrow I^{\bullet}$, we have a natural map of chain complexes $\operatorname{Tot}_{\operatorname{Hom}}\left(K^{\bullet}, G^{\bullet}\right) \rightarrow \operatorname{Tot} \operatorname{Hom}_{C}\left(K^{\bullet}, I^{\bullet}\right)$, the latter of which is a representative of $R \operatorname{Hom}_{C}\left(K^{\bullet}, G^{\bullet}\right)$, and yields the first map of the lemma, provided that the functor ind $\operatorname{Tot} \operatorname{Hom}_{C}\left(K^{\bullet}, \ldots\right)$ descends to the derived category, e.g., preserves quasi-isomorphisms. But this is easily checked, once we know it is quasi-isomorphic to $R \operatorname{Hom}_{C}\left(K^{\bullet}, G^{\bullet}\right)$. Hence the first statement (isomorphism of ind-objects in the derived category) follows from the second (isomorphism of cohomology).

To prove the second, we first reduce to the case where $G^{\bullet}=G$ is a single object concentrated in degree zero. Assume the lemma is true in this case. The results extend to complexes concentrated in a single non-zero degree by reindexing. For finite complexes, note that

$$
\operatorname{ind}_{K^{\bullet} \in h D} \operatorname{Tot} \operatorname{Hom}_{C}\left(K^{\bullet}, \ldots\right) \simeq R \operatorname{Hom}_{C}\left(X^{\bullet}, \ldots\right)
$$

take short exact sequences to exact triangles. Any finite truncation filtration with subquotients complexes concentrated in a single degree and induction gives the result for finite complexes.

For a bounded below positive complex $G^{\bullet}$, we note that

$$
\underset{n}{\lim _{\longrightarrow}} R \operatorname{Hom}_{C}\left(K^{\bullet}, G^{\leqslant n}\right) \simeq R \operatorname{Hom}_{C}\left(K^{\bullet}, \underset{n}{\lim _{\longrightarrow}} G^{\leqslant n}\right) \simeq R \operatorname{Hom}_{C}\left(K^{\bullet}, G^{\bullet}\right)
$$

if $K^{\bullet}$ is a bounded above complex: $R^{i} \operatorname{Hom}_{C}\left(K^{\bullet}, G^{\bullet}\right) \simeq R^{i} \operatorname{Hom}_{C}\left(K^{\bullet}, G^{\leqslant n}\right)$ for some $n$ sufficiently large, since the overlap between $K^{\bullet}$ and $G^{\bullet}[i]$ is finite. Likewise,

$$
\underset{n}{\lim _{K} \operatorname{ind}_{K^{\bullet} D}} \operatorname{Tot} \operatorname{Hom}_{C}\left(K^{\bullet}, G^{\leqslant n}\right) \simeq \operatorname{ind}_{K^{\bullet} \in h D} \operatorname{Tot} \operatorname{Hom}_{C}\left(K^{\bullet}, \underset{n}{\lim _{n}} G^{\leqslant n}\right) .
$$

This gives the result for bounded below complexes.

It remains to show the second statement when $G^{\bullet}=G$ is a single object concentrated in degree zero. To compute $\operatorname{RHom}\left(K^{\bullet}, G\right)$, the first quadrant double complex $\operatorname{Hom}_{C}\left(K^{\bullet}, I^{\bullet}\right)\left(\right.$ recall $\operatorname{Hom}_{C}\left(K^{a}, I^{b}\right)$ has bidegree $\left.(-a, b)\right)$ gives $\operatorname{Tot} \operatorname{Hom}_{C}\left(K^{\bullet}, I^{\bullet}\right)$ a decreasing filtration by columns

$$
F^{l} \operatorname{Tot}^{m} \operatorname{Hom}_{C}\left(K^{\bullet}, I^{\bullet}\right)=\bigoplus_{\substack{a+b=m \\ a \leqslant l}} \operatorname{Hom}_{C}\left(K^{a}, I^{b}\right) .
$$

We get a first quadrant convergent spectral sequence

$$
\begin{aligned}
E_{1}^{p, q} & =H^{q}\left(\operatorname{Hom}_{C}\left(K^{p}, I^{\bullet}\right)\right)=\operatorname{Ext}_{C}^{q}\left(K^{p}, G\right) \\
& \Rightarrow H^{p+q} \operatorname{RHom}\left(K^{\bullet}, G\right)=\operatorname{Ext}_{C}^{p+q}\left(X^{\bullet}, G\right) .
\end{aligned}
$$

The $E_{1}$ terms do not form a cofiltered system, since only $h D$ is cofiltered. However, since the $E_{2}$ terms are the horizontal cohomology of the $E_{1}$ terms and chain homotopic maps induce the same map on cohomology, we can take the filtered colimit of the $E_{2}$ terms over $h D$. This yields a colimit spectral sequence

$$
E_{2}^{p, q}=\underset{K \in h D}{\lim _{K \in}} H^{p}\left(\operatorname{Ext}_{C}^{q}\left(K^{\bullet}, G\right)\right) \Rightarrow \operatorname{Ext}_{C}^{p+q}\left(X^{\bullet}, G\right) .
$$

The objects on the left-hand side are cohomologies of the complexes of Ext $_{C}^{q}$ by varying the $K^{\bullet}$. By property 2 of $D$, and the following well-known lemma, all $\gamma \in$ Ext $^{q}$ 
with $q>0$ vanish in the limit, collapsing the spectral sequence at the $E_{2}$ page and yielding the desired isomorphism

$$
\underset{K \in h D}{\lim _{K \in}} H^{p}\left(\operatorname{Hom}_{C}\left(K^{\bullet}, G\right)\right)=\operatorname{Ext}_{C}^{p}\left(X^{\bullet}, G\right) .
$$

Lemma 2.8. Let $C$ be an abelian category with enough injectives. For any $K, A \in C$, any $q>0$ and any extension class

$$
\gamma \in \operatorname{Ext}_{C}^{q}(K, A)
$$

there is an epimorphism $f: M \rightarrow K$ so that $f^{*}(\gamma)=0$ in $\operatorname{Ext}_{C}^{q}(M, A)$.

Proof. For a fixed $q>0$, choose a truncated injective resolution

$$
0 \rightarrow A \rightarrow I^{0} \rightarrow \cdots \rightarrow I^{q-1} \rightarrow J \rightarrow 0,
$$

where $J$ is the cokernel of $I^{q-2} \rightarrow I^{q-1}$. Applying the functor $\operatorname{Hom}(K, \ldots)$ yields the complex

$$
\operatorname{Hom}\left(K, I^{0}\right) \rightarrow \cdots \rightarrow \operatorname{Hom}\left(K, I^{q-1}\right) \rightarrow \operatorname{Hom}(K, J) \rightarrow \operatorname{Ext}^{q}(K, A) \rightarrow 0 .
$$

Lift $\gamma$ to a homomorphism $\sigma: K \rightarrow J$ in $\operatorname{Hom}(K, J)$, and form the fiber product $I^{q-1} \times{ }_{J} K$ using $\sigma$. The natural projection map $f: M=I^{q-1} \times{ }_{K} K \rightarrow K$ yields a map of complexes

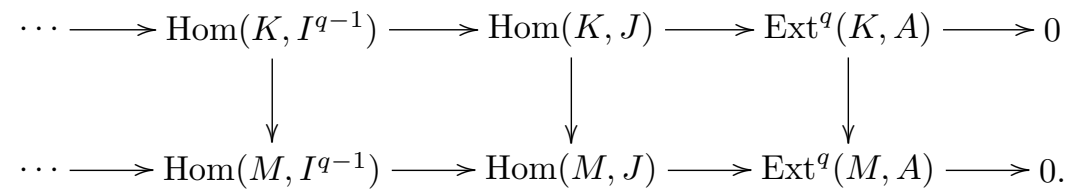

By construction, $f^{*}(\sigma) \in \operatorname{Hom}(M, J)$ is $u \circ p_{2}$ in the cartesian square

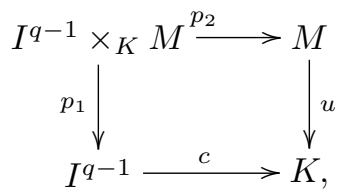

but $u \circ p_{2}=c \circ p_{1}$ is the image of $p_{1} \in \operatorname{Hom}\left(M, I^{q-1}\right)$. Hence $f^{*}(\sigma)$ is a coboundary and so $f^{*}(\gamma)$ is zero.

\subsection{Main theorem}

Recall that $C$ is a site, possibly without finite products and fiber products.

Definition 2.9. For a fixed simplicial presheaf $X_{\bullet} \in s$ Pre $C$, let $D\left(X_{\bullet}\right)$ denote the subcategory of $s \operatorname{Pre} C / X_{\bullet}$ of local acyclic fibrations $K_{\bullet} \rightarrow X_{\bullet}$.

For any category of simplicial objects $E$, write $h E$ to be the same category with morphisms up to simplicial homotopy. In general, this is not an equivalence relation; we use the relation generated by simplicial homotopy.

Proposition 2.10 (cf. [12, ex V Theorem 7.3.2]). Let $X_{\bullet} \in s \operatorname{Pre} C$ be a simplicial presheaf. Then: 
1. The homotopy category $h D\left(X_{\bullet}\right)$ is cofiltered.

2. For every $K_{\bullet} \in h D\left(X_{\bullet}\right)$, object $M \in C$, and covering morphism $u: M \rightarrow K_{n}$, there is an object $L_{\bullet} \in h D\left(X_{\bullet}\right)$ and a morphism $f: L_{\bullet} \rightarrow K_{\bullet}$ whose degree $n$ part factors as

$$
f_{n}: L_{n} \rightarrow M \stackrel{u}{\rightarrow} K_{n}
$$

3. For every $K_{\bullet} \in D\left(X_{\bullet}\right)$, the structure morphism $K_{\bullet} \rightarrow X_{\bullet}$ induces a quasi-isomorphism

$$
\mathbb{Z} K^{\bullet} \rightarrow \mathbb{Z} X^{\bullet}
$$

Proof of Part 3 of Proposition 2.10. This is just Proposition 2.5.

Proof of Part 2 of Proposition 2.10. The following proof is mostly unchanged from Verdier's original: Let $j_{n *}$ be the right adjoint of "taking the degree $n$ component". We claim $j_{n *}$ takes covering morphisms to local acyclic fibrations. Let $f: A \rightarrow B$ be a covering morphism of presheaves. Then we must check, for an open $U \in C$, that we can locally lift a diagonal in a diagram

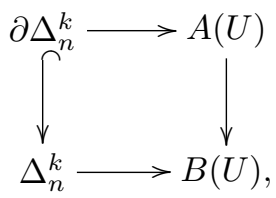

but since $A \rightarrow B$ is a covering, it is a surjection after a refinement $V$ of $U$, so we can always lift $\Delta_{n}^{k} \rightarrow A(V)$.

To prove Part 2, form the cartesian diagram

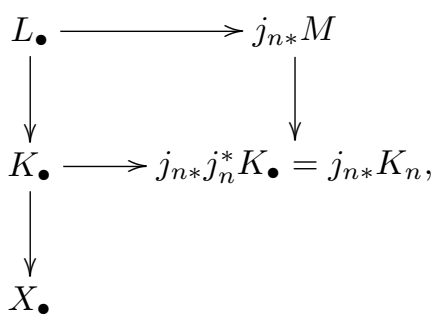

where the right vertical arrow is given by functoriality and the bottom horizontal arrow is given by adjunction. The right vertical arrow is a local acyclic fibration by the above remark. By Lemma 2.6, $L_{\bullet} \rightarrow K_{\bullet} \rightarrow X_{\bullet}$ is a local acyclic fibration, and $L_{n} \rightarrow K_{n}$ factors as $L_{n} \rightarrow M \rightarrow K_{n}$.

Proof of Part 1 of Proposition 2.10. (We warn the reader that there is a small, inconsequential error in Verdier's original.) Suppose we are given a diagram

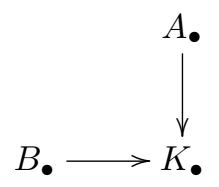

in $D\left(X_{\bullet}\right)$. Set $L_{\bullet}=A_{\bullet} \times_{X_{\bullet}} B_{\bullet}$ which exists in $s$ Pre $C$. Lemma 2.6 shows that the 
canonical map $L_{\bullet} \rightarrow X_{\bullet}$ is a local acyclic fibration, so it is in $D\left(X_{\bullet}\right)$. This gives a possibly non-commutative diagram

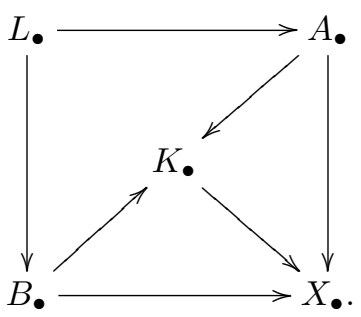

Hence we have two maps $L_{\bullet} \rightrightarrows K_{\bullet}$ which we wish to equalize up to homotopy. Thus, to prove $h D\left(X_{\bullet}\right)$ is cofiltered, it is enough to show that for every pair of morphisms in $D\left(X_{\bullet}\right)$,

$$
L \bullet \underset{u_{1}}{\stackrel{u_{0}}{\rightrightarrows}} K_{\bullet}
$$

there is a morphism $v: M_{\bullet} \rightarrow L_{\bullet}$ in $D\left(X_{\bullet}\right)$ so that the two morphisms $u_{0} v$ and $u_{1} v$ are homotopic. That is, there are commutative diagrams

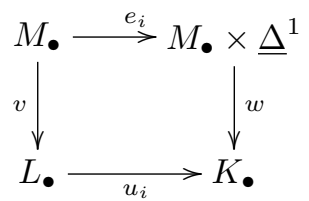

for $i=0,1$, where the $e_{i}$ are the standard inclusions, and $w$ is the homotopy.

The set of such diagrams for fixed $M_{\bullet}$ and $L_{\bullet} \underset{u_{1}}{\stackrel{u_{0}}{\rightrightarrows}} K_{\bullet}$ is given by

$$
\operatorname{Hom}\left(M_{\bullet} \times \underline{\Delta}^{1}, K_{\bullet}\right) \times_{\operatorname{Hom}\left(M_{\bullet}, K_{\bullet} \times K_{\bullet}\right)} \operatorname{Hom}\left(M_{\bullet}, L_{\bullet}\right),
$$

where the map from $\operatorname{Hom}\left(M_{\bullet}, L_{\bullet}\right)$ to $\operatorname{Hom}\left(M_{\bullet}, K_{\bullet} \times K_{\bullet}\right)$ is induced from $u_{1} \times u_{2}$, and the map $\operatorname{Hom}\left(M_{\bullet} \times \underline{\Delta}^{1}, K_{\bullet}\right)$ to $\operatorname{Hom}\left(M_{\bullet}, K_{\bullet} \times K_{\bullet}\right)$ is induced from $e_{0} \times e_{1}$.

The functor

$$
\operatorname{Hom}\left(\ldots \times \underline{\Delta}^{1}, K_{\bullet}\right) \times_{\operatorname{Hom}\left(\ldots, K_{\bullet} \times K_{\bullet}\right)} \operatorname{Hom}\left(\ldots, L_{\bullet}\right)
$$

is equal to

$$
\begin{aligned}
\operatorname{Hom}\left(\ldots, \operatorname{sHom}\left(\underline{\Delta}^{1}, K_{\bullet}\right)\right) \times & \times_{\operatorname{Hom}\left(\ldots, K_{\bullet} \times K_{\bullet}\right)} \operatorname{Hom}\left(\ldots, L_{\bullet}\right) \\
& =\operatorname{Hom}\left(\ldots, \operatorname{som} o m\left(\underline{\Delta}^{1}, K_{\bullet}\right) \times_{K_{\bullet} \times K_{\bullet}} L_{\bullet}\right)
\end{aligned}
$$

and so is representable. This representing object will be our $M_{\bullet}$. We must show that 
$M_{\bullet} \rightarrow X_{\bullet}$ is a local acyclic fibration. $M$ is the pullback in the square in the diagram

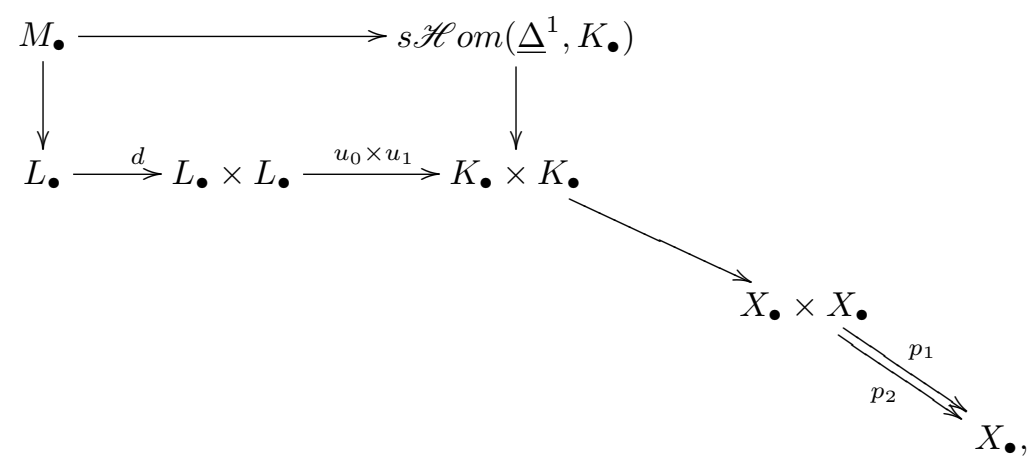

where $d$ is the diagonal. Note all maps to $X \bullet$ are the same, and

$$
K_{\bullet} \times K_{\bullet}=\operatorname{sH} o m\left(\underline{\partial \Delta}^{1}, K_{\bullet}\right) .
$$

Thus, to lift a diagram

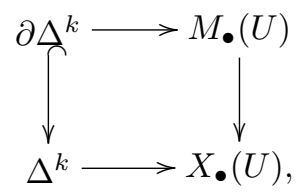

we have to lift from $X_{\bullet}$ to $L_{\bullet}, K_{\bullet} \times K_{\bullet}$ and $s \mathscr{H} o m\left(\underline{\Delta}^{1}, K_{\bullet}\right)$ with the following compatibility condition: the lift $\Delta^{k} \rightarrow L_{\bullet}(V)$ yields by composition with the diagonal a lift $\Delta^{k} \rightarrow L_{\bullet}(V) \times L_{\bullet}(V)$, or a map $\Delta^{k} \times \partial \Delta^{1} \rightarrow L_{\bullet}(V)$. By composition with the map $u_{0} \times u_{1}$, we get a lift $\Delta^{k} \times \partial \Delta^{1} \rightarrow K_{\bullet}(V)$. Meanwhile a lift to $\operatorname{sH} o m\left(\Delta^{1}, K_{\bullet}\right)$ is a map $\Delta^{k} \times \Delta^{1} \rightarrow K(V)$, which by pre-composition with the inclusion $\partial \Delta^{1} \subset \Delta^{1}$ yields a map $\Delta^{k} \times \partial \Delta^{1} \rightarrow K(V)$. We require these two maps to be equal.

But we can guarantee this as follows: giving the lifting diagram above, we extend by projection to the first factor to a diagram

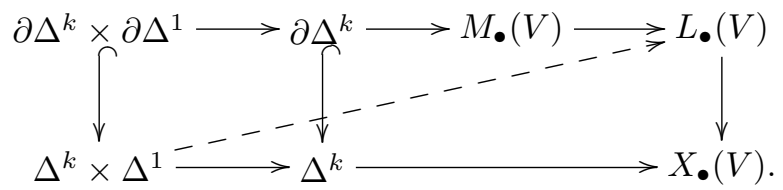

By Lemma 2.2, we can lift to get the dashed arrow. This yields a composition

$$
\Delta^{k} \times \partial \Delta^{1} \rightarrow \Delta^{k} \times \Delta^{1} \rightarrow L_{\bullet}(V) \rightarrow K_{\bullet}(V),
$$

e.g., lifts to $L_{\bullet}(V) \times L_{\bullet}(V)$ and $\operatorname{sH} o m\left(\underline{\Delta}^{1}, K_{\bullet}\right)(V)$, which map to the same the lift to $K_{\bullet}(V) \times K_{\bullet}(V)$. The compatibility of the maps to $X_{\bullet}$, and the fact that $L_{\bullet} \rightarrow$ $L_{\bullet} \times L_{\bullet}$ is the diagonal, ensures that these lifts are compatible with the maps in the fiber product.

Fix a $X_{\bullet} \in s \operatorname{Pre} C$. Let $\operatorname{Ab}(\operatorname{Sh} C)$ be the category of sheaves of abelian groups on $C$. A simplicial presheaf $K_{\bullet}$ yields a negative cochain complex of sheaves of free abelian groups $\mathbb{Z} K^{\bullet}$. We abuse notation and also call $D\left(X_{\bullet}\right)$ the image of $D\left(X_{\bullet}\right)$ 
inside $\mathrm{Ch}^{-}(\mathrm{Ab}(\mathrm{Sh} C))$ under this functor. Note that simplicial homotopy of simplicial presheaves becomes chain homotopy of cochain complexes under this functor.

Our basic result on hyper-Čech cohomology is

Theorem 2.11. Let $X_{\bullet}$ be a simplicial presheaf, and $h D\left(X_{\bullet}\right)$ the homotopy category of local acyclic fibrations $K_{\bullet} \rightarrow X_{\bullet}$. Then for a bounded below complex of sheaves of abelian groups $\mathscr{F} \bullet$ with the filtration bête

$$
{\underset{K}{\bullet} \in \overrightarrow{h D}(X \bullet)}_{\longrightarrow} H^{p}\left(\operatorname{Tot} \operatorname{Hom}\left(\mathbb{Z} K^{\bullet}, \mathscr{F} \bullet\right)\right) \simeq \operatorname{Ext}^{p}\left(\mathbb{Z} X^{\bullet}, \mathscr{F} \bullet\right)
$$

and there is a filtered quasi-isomorphism of ind-objects in the derived category

$$
\operatorname{ind}_{K \bullet \in h D\left(X_{\bullet}\right)} \operatorname{Tot} \operatorname{Hom}\left(\mathbb{Z} K^{\bullet}, \mathscr{F} \bullet\right) R \operatorname{Hom}\left(\mathbb{Z} X^{\bullet}, \mathscr{F} \bullet\right) .
$$

Proof. According to Proposition 2.10, $D\left(X_{\bullet}\right)$ is a subcategory of $\mathrm{Ch}^{-}(\mathrm{Ab}(\mathrm{Sh} C))$ which satisfies the properties of the Lemma 2.7, the lemma on computing Ext, which gives the result.

\subsection{Semi-representability and para-representability}

Definition 2.12. A presheaf is semi-representable if it is isomorphic to a coproduct of representable presheaves. A presheaf is para-representable if it is isomorphic to a finite coproduct of representable presheaves. A simplicial presheaf is semi-representable (resp. para-representable) if all of its components are.

The theorems above show that representability hypotheses are not important in the computation of sheaf cohomology. However, typically one wishes to compute with representable or semi-representable presheaves. For this we have

Lemma 2.13 (A Godement-type lemma). Any presheaf is covered by a semi-representable presheaf.

Proof. For a presheaf $F$, we have the presheaf surjection

$$
\coprod_{(X \in C, s \in F(X))} h_{X} \rightarrow F
$$

where $h_{X}$ denotes the representable presheaf given by $\operatorname{Hom}(\ldots, X)$. Since $\operatorname{Hom}(X, F)$ $=F(X)$, the morphism is given by $s$. This is obviously surjective on the level of sets, and since sheafification is exact, it is a covering.

Remark 2.14. We make use of the formalism of split simplicial objects, cf. [12, ex Vbis $5.1]$ or $[4,6.2 .2]$, which allows us to construct semi-representable simplicial presheaves inductively by only specifying the non-degenerate pieces. The degeneracies are satisfied by adding copies of the lower degree pieces; all maps between such objects are isomorphisms, so will satisfy whatever requirements we have of them (properness, coverings, et cetera) and will come equipped inductively via the degeneracies with maps to any desired target.

Proposition 2.15. Let $S R\left(X_{\bullet}\right)$ be the full subcategory of $D\left(X_{\bullet}\right)$ of objects whose components are semi-representable; then $h S R\left(X_{\bullet}\right)$ is a cofinal subcategory of $h D\left(X_{\bullet}\right)$. 
Thus, for a bounded below complex of sheaves of abelian groups $\mathscr{F}^{\bullet}$ with the filtration bête

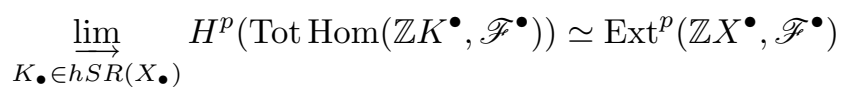

and there is a filtered quasi-isomorphism of ind-objects in the derived category

$$
\operatorname{ind}_{K \bullet \in h S R\left(X_{\bullet}\right)} \operatorname{Tot} \operatorname{Hom}\left(\mathbb{Z} K^{\bullet}, \mathscr{F} \bullet\right) \simeq R \operatorname{Hom}\left(\mathbb{Z} X^{\bullet}, \mathscr{F} \bullet\right)
$$

Proof. It is enough to show, for any local acyclic fibration $K_{\bullet} \rightarrow X_{\bullet}$, there is a local acyclic fibration $L_{\bullet} \rightarrow K_{\bullet}$ with $L_{\bullet}$ semi-representable. We construct one inductively as follows: set $L_{0} \rightarrow K_{0}$ a semi-representable cover given by the Godement lemma. Having constructed $L$ • to degree $i-1$, set

$$
L^{\prime} \rightarrow\left(i_{i-1 *} L\right)_{i} \times\left(\operatorname{cosk}_{i-1} K\right)_{i} K_{i}
$$

to be a semi-representable cover given by the Godement lemma. We set $L_{i}$ to be the union of $L^{\prime}$ and the copies of the $L_{k}$ for $k<i$ needed to satisfy the degeneracy relations; see Remark 2.14 .

Remark 2.16. This gives a generalized version of Verdier's theorem on hypercovers ([12, ex V Theorem 7.4.1]).

On sites without finite products and fiber products, we need some additional hypotheses for para-representability. The following result will be useful in the application to $\mathrm{Sm}_{h} \subset \mathrm{Sch}_{h}$; cf. Corollary 3.18.

Proposition 2.17. Suppose the site $C$ is a full subcategory of a larger site $C^{\prime}$, and

1. The topology on $C^{\prime}$ is generated by a pretopology all of whose covering families are finite.

2. C has the induced topology.

3. $C^{\prime}$ has finite products and fiber products.

4. Every $Y \in C^{\prime}$ can be covered by an $X \in C$.

Let $X_{\bullet} \in s$ Pre $C^{\prime}$ be a para-representable simplicial presheaf. Let $F R_{C}\left(X_{\bullet}\right)$ be the subcategory of

$$
S R_{C^{\prime}}\left(X_{\bullet}\right)=\left\{\text { semi-representable local acyclic fibrations in } C^{\prime}\right\}
$$

whose components are para-representable and in $C$. Then $h F R_{C}\left(X_{\bullet}\right)$ is cofinal in $h S R_{C^{\prime}}\left(X_{\bullet}\right)$. Thus, for a bounded below complex of sheaves of abelian groups $\mathscr{F} \bullet$ with the filtration bête

$$
{\underset{K}{\bullet} \in h \overrightarrow{\lim }_{C}\left(X_{\bullet}\right)} H^{p}\left(\operatorname{Tot} \operatorname{Hom}\left(\mathbb{Z} K^{\bullet}, \mathscr{F}^{\bullet}\right)\right) \simeq \operatorname{Ext}^{p}\left(\mathbb{Z} X^{\bullet}, \mathscr{F}^{\bullet}\right)
$$

and there is a filtered quasi-isomorphism of ind-objects in the derived category

$$
\operatorname{ind}_{K_{\bullet} \in h F R_{C}\left(X_{\bullet}\right)} \operatorname{Tot} \operatorname{Hom}\left(\mathbb{Z} K^{\bullet}, \mathscr{F}^{\bullet}\right) \simeq R \operatorname{Hom}\left(\mathbb{Z} X^{\bullet}, \mathscr{F}^{\bullet}\right) .
$$

Proof. The hypotheses on $C$ and $C^{\prime}$ show that: 
1. Every covering morphism $F \rightarrow G$ in $C^{\prime}$ where $F$ is semi-representable and $G$ is para-representable can be refined $E \rightarrow F \rightarrow G$, where $E$ is para-representable in $C$ and $E \rightarrow G$ is a covering morphism.

2. Finite limits of para-representable presheaves in $C^{\prime}$ can be covered by pararepresentable presheaves in $C$.

By Verdier's theorem it is enough to show, for every semi-representable local acyclic fibration $K_{\bullet} \rightarrow X_{\bullet}$ in $C^{\prime}$, there is a para-representable local acyclic fibration $L_{\bullet} \rightarrow X_{\bullet}$ with $L_{\bullet}$ in $C$ and a map over $X_{\bullet}$ of simplicial presheaves $L_{\bullet} \rightarrow K_{\bullet}$. Set $L_{0} \subset K_{0}$ a subpresheaf which is para-representable in $C$ and covers $X_{0}$. Suppose inductively we have constructed $L$ • to degree $i-1$. Then $\left(i_{i-1 *} L\right)_{i} \times\left(\operatorname{cosk}_{i-1} X\right)_{i} X_{i}$ is a finite limit of para-representable presheaves, so cover it with $L^{\prime}$ a para-representable in $C$. Construct the fiber product $L^{\prime} \times\left(\operatorname{cosk}_{i-1} K\right)_{i} K_{i}$, cover it with a semi-representable $L^{\prime \prime}$. Then $L^{\prime \prime} \rightarrow L^{\prime}$ is a cover of a para-representable by a semi-representable, so take $L^{\prime \prime \prime} \rightarrow L^{\prime \prime} \rightarrow L^{\prime}$ with $L^{\prime \prime \prime}$ para-representable in $C$ and $L^{\prime \prime \prime} \rightarrow L^{\prime}$ a cover. As before, we have to add copies of $L_{k}$ for $k<i$ to satisfy degeneracy conditions; cf. Remark 2.14. By construction, there is a map $L_{\bullet} \rightarrow K_{\bullet}$ and the composite $L_{\bullet} \rightarrow X_{\bullet}$ is a local acyclic fibration.

\section{3. $h$ - and $u c d$-topologies}

\subsection{The $h$-topology}

Definition 3.1. A $\mathbb{C}$-scheme is a separated scheme finite type over the field of complex numbers. Let Sch denote the category of $\mathbb{C}$-schemes, and let $\mathrm{Sm} \subset \mathrm{Sch}$ denote the full subcategory of smooth $\mathbb{C}$-schemes. If $X \in \mathrm{Sch}$, let $\mathrm{Sch} / X, \mathrm{Sm} / X$ denote the categories of $\mathbb{C}$-schemes and smooth $\mathbb{C}$-schemes over $X$.

We recall Voevodsky's ([23]) $h$-topology:

Definition 3.2. A morphism $f: X \rightarrow Y$ is called a topological epimorphism if the underlying morphism of topological spaces is a topological quotient map: it is surjective on sets and $U \subset Y$ is open if and only if $f^{-1}(U)$ is open in $X$. A universal topological epimorphism, or an $h$-covering, is a morphism $X \rightarrow Y$ so that for any $Z \rightarrow Y$, the base change morphism

$$
X \times{ }_{Z} Y \rightarrow Z
$$

is a topological epimorphism.

A useful necessary but not sufficient characterization of $h$-coverings is given by the following:

Proposition 3.3 ([23, Proposition 3.1.3]). Let $f: X \rightarrow Y$ be a morphism of schemes, and $X^{\prime} \subset X$ the union of the irreducible components of $X$ which dominates some component of $Y$. If $f$ is an h-covering, then $f\left(X^{\prime}\right)=Y$.

Definition 3.4. The $h$-topology is the topology on Sch induced from the pretopology given by finite families $\left\{U_{i} \rightarrow X\right\}$, where $\amalg U_{i} \rightarrow X$ is an $h$-covering. We denote the site of $\mathbb{C}$-schemes with the $h$-topology $\operatorname{Sch}_{h}$. Sm inherits a topology from $\operatorname{Sch}_{h}$ as in [11, ex III 3.1]; by resolution of singularities $([2,17])$, this topology is just given by restricting covering sieves of $\mathrm{Sch}_{h}$ to $\mathrm{Sm}$; we denote this site $\mathrm{Sm}_{h}$. 
Remark 3.5. Note that, by resolution of singularities ([2,17]), $\operatorname{Sm}_{h} \subset \operatorname{Sch}_{h}$ satisfy the conditions of Proposition 2.17, so para-representable hypercovers compute sheaf cohomology in $\mathrm{Sm}_{h}$.

Theorem 3.6 ([11, ex III Theorem 4.1]). Let $C, C^{\prime}$ be small categories, and let $u: C \rightarrow C^{\prime}$ be a fully faithful functor. Suppose $C^{\prime}$ has a Grothendieck topology, and let $C$ have the induced topology. If every object of $C^{\prime}$ can be covered by an object of $C$, then the functor $F \mapsto F \circ u$ is an equivalence of the category of sheaves on $C^{\prime}$ with the category of sheaves on $C$.

Corollary 3.7. The category of sheaves on $\mathrm{Sm}_{h}$ is equivalent via the natural embedding to the category of sheaves on $\mathrm{Sch}_{h}$.

Proof. Resolution of singularities $([2,17])$ gives smooth $h$-coverings of arbitrary $\mathbb{C}$ schemes.

\subsection{Cohomological descent}

Definition 3.8 (Cohomological descent). An augmented simplicial $\mathbb{C}$-scheme

$$
e: K \bullet \rightarrow X
$$

is a cohomological descent resolution if the adjunction

$$
\operatorname{id}_{a n} \rightarrow R e_{a n *} e_{a n}^{*}
$$

is an isomorphism; here we use the analytic topology. According to [13, ex XVI 4.1], if one restricts to rational vector spaces, then this is the same as requiring $\mathbb{Q}_{l, X} \simeq R e_{*}\left(\mathbb{Q}_{l, K_{\bullet}}\right)$ in the étale topology. The morphism $e$ is a universal cohomological descent resolution (or a ucd-resolution) if it is a cohomological descent resolution after any base change.

A morphism of $\mathbb{C}$-schemes $Y \rightarrow X$ is of cohomological descent if $\operatorname{cosk}_{0}(Y / X) \rightarrow X$ is a cohomological descent resolution (where $\operatorname{cosk}_{0}(Y / X)$ is the coskeleton functor in the category of schemes over $X$ ). A morphism $Y \rightarrow X$ is universally of cohomological descent (or a ucd-cover) if every base change is of cohomological descent.

Some basic results:

Lemma $3.9([4,5.3 .5])$. A morphism with a local section is a ucd-covering. A proper surjection is a ucd-covering.

Lemma 3.10 ([4, 5.3.5]).

1. The composition of ucd-coverings is a ucd-covering.

2. If the composition $X \rightarrow Y \stackrel{f}{\rightarrow} Z$ is a ucd-covering, then $f$ is a ucd-covering.

Proof. See [1, Theorem 7.5] for a proof.

According to $[4,5.3 .5]$, ucd-coverings form a pretopology on Sch. We deviate from Deligne, however, in taking the pretopology generated by only finite families $\left\{U_{i} \rightarrow X\right\}$, where $\amalg U_{i} \rightarrow X$ is a $u c d$-covering. (Deligne and Du Bois in practice use only representable simplicial objects so there is no difference.) We denote the topol- 
ogy generated by this pretopology as the universal cohomological descent topology, or the ucd-topology.

Let $\mathrm{Sch}_{u c d}$ be the category of $\mathbb{C}$-schemes with the $u c d$-topology. Since resolution of singularities are $u c d$-coverings, by the exact same argument as for the $h$-topology, the induced topology on $\mathrm{Sm}$ (denoted $\mathrm{Sm}_{u c d}$ ) is given by restricting the covering sieves of $\mathrm{Sch}_{u c d}$, and the categories of sheaves on $\mathrm{Sch}_{u c d}$ and $\mathrm{Sm}_{u c d}$ are equivalent.

Remark 3.11. Again $\mathrm{Sch}_{u c d}$ and $\mathrm{Sm}_{u c d}$ satisfy the conditions of Proposition 2.17, so para-representable hypercovers compute sheaf cohomology in $\mathrm{Sm}_{u c d}$.

The basic, almost circular theorem is

Theorem $3.12([4,5.3 .5])$. Let $e: K_{\bullet} \rightarrow X$ be a hypercover in the topology of universal cohomological descent. Then $f$ is a universal cohomological descent resolution.

Remark 3.13. Note that both the $h$-topology and the $u c d$-topology refer to an underlying topology: the $h$-topology refers to the Zariski topology, and the $u c d$-topology refers to the étale or analytic topologies.

\subsection{Comparison of the $h$ - and $u c d$-topologies}

Lemma 3.14 ([23, Theorem 3.1.9]). An h-covering $Y \rightarrow X$ of an excellent reduced noetherian scheme $X$ can be refined $Y^{\prime} \rightarrow Y \rightarrow X$ to an $h$-covering of normal form: $Y^{\prime} \rightarrow X$ factors as $s \circ f \circ i$, where $i$ is an open covering, $f$ is a finite surjective morphism, and $s$ is a blowup of a closed subscheme.

Corollary 3.15. An h-covering $Y \rightarrow X$ in $\mathrm{Sm}_{h}$ can be refined to $Y^{\prime} \rightarrow Y \rightarrow X$, where $Y^{\prime} \rightarrow X$ factors into $Y^{\prime} \rightarrow Z \rightarrow X$, where $Y^{\prime} \rightarrow Z$ is a Zariski open cover, $Z \rightarrow X$ is proper, and $Y^{\prime}$ and $Z$ are smooth. Moreover, we may assume that both $Y^{\prime}$ and $Z$ are quasi-projective.

Proof. $\mathbb{C}$-schemes are excellent. Factor $Y \rightarrow X$ to $Y^{\prime \prime} \rightarrow Y \rightarrow X$ with $Y^{\prime \prime} \rightarrow Z^{\prime} \rightarrow X$, where $Y^{\prime \prime} \rightarrow Z^{\prime}$ is a Zariski open cover and $Z^{\prime} \rightarrow X$ is proper (composition of a finite morphism and a blowup). Use resolution of singularities to get $Z \rightarrow Z^{\prime} \rightarrow X$ proper, take $Y^{\prime}=Y^{\prime \prime} \times_{Z^{\prime}} Z$, which will be a Zariski open cover of $Z$.

To get the last statement, use Chow's lemma [14, 5.6.1, 5.6.2] to get $Z^{\prime} \rightarrow Z$ by a projective surjective morphism with $Z^{\prime}$ quasi-projective, and the base change $Y^{\prime \prime}=Y^{\prime} \times{ }_{Z} Z^{\prime}$ is a Zariski open cover of $Z^{\prime}$.

Corollary 3.16. An h-covering in $\mathrm{Sch}$ or $\mathrm{Sm}$ is a ucd-covering.

Proof. By the lemma or the corollary, an $h$-covering $f: Y \rightarrow X$ in either Sch or Sm has a refinement $Y^{\prime} \stackrel{g}{\rightarrow} Y \stackrel{f}{\rightarrow} X$ in either Sch or Sm, where $f \circ g$ factors into a composition of morphisms which are universally of cohomological descent. Hence by Lemma 3.10, $f$ is universally of cohomological descent.

By the above proposition, we have continuous functors

$$
\mathrm{Sch}_{h} \rightarrow \mathrm{Sch}_{u c d} \quad \text { and } \quad \mathrm{Sm}_{h} \rightarrow \mathrm{Sm}_{u c d}
$$

(see [11, ex III Proposition 1.6]) and thus geometric morphisms of their associated topoi of sheaves. We do not know of an example of a $u c d$-covering which is not an $h$-covering. 


\subsection{Representable hypercovers in the $h$ - or $u c d$-topologies}

Lemma 3.17. If $L$ • is a para-representable hypercover in either $\mathrm{Sm}_{h}, \mathrm{Sch}_{h}, \mathrm{Sm}_{u c d}$ or $\mathrm{Sch}_{u c d}$, then there is a representable hypercover $K_{\bullet}$ in the same site and a morphism $L_{\bullet} \rightarrow K_{\bullet}$ so that $\mathbb{Z} L^{\bullet}$ is quasi-isomorphic to $\mathbb{Z} K^{\bullet}$.

Proof. For a finite family $\left\{U_{i}\right\}$,

$$
\coprod \operatorname{Hom}\left(\ldots, U_{i}\right) \rightarrow \operatorname{Hom}\left(\ldots, \coprod U_{i}\right)
$$

is a Zariski cover, so in particular it is an $h$ - and $u c d$-cover. Thus they have the same associated sheaves of abelian groups.

In addition, if

$$
\coprod \operatorname{Hom}\left(\ldots, U_{i}\right) \rightarrow \coprod \operatorname{Hom}\left(\ldots, V_{j}\right)
$$

is a morphism of para-representable presheaves, then Yoneda's lemma tells us that the identity morphisms $\operatorname{id}_{U_{i}} \in \operatorname{Hom}\left(U_{i}, U_{i}\right)$ determine the diagonal in the commutative diagram

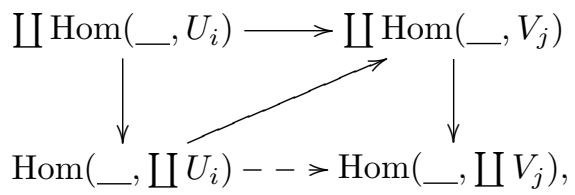

and thus the dashed arrow. Hence every morphism of para-representable presheaves determines a morphism of associated representable coproducts (but not vice versa!) and these morphisms are the same on passing to associated sheaves.

Thus, given a para-representable hypercover $L_{\bullet}$ with $L_{n}=\coprod \operatorname{Hom}\left(\ldots, U_{n, i}\right)$, take $K \bullet$ with $K_{n}=\operatorname{Hom}\left(\ldots, \amalg U_{n, i}\right)$ with simplicial morphisms given as above. It is representable and yields the same complex of sheaves of abelian groups (it is in fact a local acyclic fibration in the Zariski topology, since locally it has sections).

Corollary 3.18. Let $X_{\bullet}$ be a representable simplicial presheaf in $\operatorname{Sch}_{h}$. Let $R_{\mathrm{Sm}}\left(X_{\bullet}\right)$ be the subcategory of $F R_{\mathrm{Sm}}\left(X_{\bullet}\right)=$ \{para-representable local acyclic fibrations in $\left.\mathrm{Sm}_{h}\right\}$ whose components are representable. Then every $L_{\bullet} \in F R_{\mathrm{Sm}}\left(X_{\bullet}\right)$ has a quasi-isomorphism $\mathbb{Z} L^{\bullet} \rightarrow \mathbb{Z} K^{\bullet}$ for some $K_{\bullet} \in R_{\mathrm{Sm}}\left(X_{\bullet}\right)$, so for a bounded below complex of sheaves of abelian groups $\mathscr{F} \bullet$ with the filtration bête

$$
\lim _{K \bullet \in h \overrightarrow{\mathrm{Sm}}_{\left(X_{\bullet}\right)}} H^{p}\left(\operatorname{Tot} \operatorname{Hom}\left(\mathbb{Z} K^{\bullet}, \mathscr{F} \bullet\right) \simeq \operatorname{Ext}^{p}\left(\mathbb{Z} X^{\bullet}, \mathscr{F} \bullet\right)\right.
$$

and there is a filtered quasi-isomorphism of ind-objects in the derived category

$$
\operatorname{ind}_{K \bullet \in h R_{\mathrm{Sm}}\left(X_{\bullet}\right)} \operatorname{Tot} \operatorname{Hom}\left(\mathbb{Z} K^{\bullet}, \mathscr{F}^{\bullet}\right) \simeq R \operatorname{Hom}\left(\mathbb{Z} X^{\bullet}, \mathscr{F} \bullet .\right.
$$

Proof. Proposition 2.17 says we may compute using para-representable hypercovers in $\mathrm{Sm}_{h}$. The lemma says para-representable hypercovers have associated complexes of sheaves of free abelian groups equivalent to those of representable hypercovers. 


\section{Algebraic de Rham complex}

\section{1. $\Omega^{q}$ is an $h$-sheaf}

For every $q \geqslant 0$, let $\Omega^{q}$ denote the presheaf on the site $\mathrm{Sm}_{h}$ given by

$$
X \mapsto \Gamma\left(X, \Omega_{X / \mathbb{C}}^{q}\right) .
$$

It is a presheaf of $\mathscr{O}$-modules.

Lemma 4.1. If $f: X \rightarrow Y$ is a dominant morphism of smooth $\mathbb{C}$-schemes, then

$$
\Omega^{q}(Y) \hookrightarrow \Omega^{q}(X) .
$$

Proof. Suppose $\omega \in \Omega^{q}(Y)$ has $f^{*} \omega=0$. Generic smoothness gives a Zariski open dense $U \subset Y, V=f^{-1}(U) \subset X$, where $\left.f\right|_{V}$ is smooth. Then $\left.f\right|_{V} ^{*}$ is injective so we see $\omega$ vanishes on an open dense set, so $\omega$ must be zero.

Proposition 4.2. $\Omega^{q}$ is a sheaf in $\mathrm{Sm}_{h}$.

Proof. We must check, for every covering sieve $R$ of $X$, that $\Omega^{q}(R)=\Omega^{q}(X)$. We may assume that $X$ is irreducible. It is enough to check for $R$ generated by a single $h$-covering family, and in fact a single covering $u: Y \rightarrow X:$ if $\left\{U_{i} \rightarrow X\right\}$ is a finite covering family, then $\Omega^{q}\left(\left\{U_{i} \rightarrow X\right\}\right)=\Omega^{q}\left(\coprod_{j} U_{j}\right)$ because $\Omega^{q}$ is already a Zariski sheaf, and $U_{i} \rightarrow \coprod_{j} U_{j}$ is a Zariski covering. Since every $f \in R$ factors through $u$, the $R$-local sections are just elements $\omega \in \Omega^{q}(Y)$ which, for every pair of maps $f, g: Z \rightrightarrows$ $Y$ with $u f=u g$, we have $f^{*} \omega=g^{*} \omega$.

We first check the case where $u$ is a smooth morphism. In this case all pairs $f, g$ factor through the smooth $W=Y \times_{X} Y \rightrightarrows Y$, so it is enough to check for $Z=W$. For $q=0$, this is the usual exact sequence of algebras

$$
0 \rightarrow A \rightarrow B \rightarrow B \otimes_{A} B
$$

where $A \hookrightarrow B$ is the injective map coming from a dominant morphism. For $q=1$ we have from the usual exact sequences of differentials the diagram

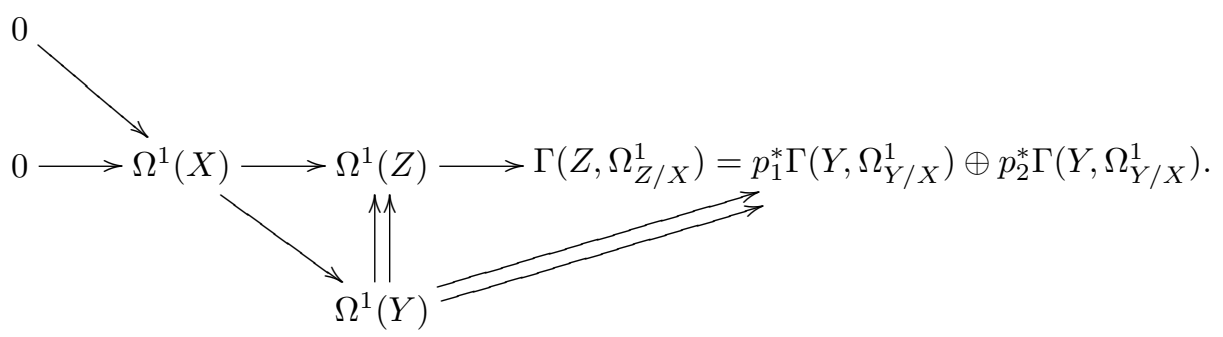

Thus $\Omega^{1}(X) \hookrightarrow \Omega^{1}(Y)$, and clearly the image is contained in the equalizer of the two vertical arrows. Conversely, if a form $\omega \in \Omega^{1}(Y)$ is sent by both vertical arrows to $\eta \in \Omega^{1}(Z)$, then commutativity of the right triangle gives that $\omega$ must be sent to the same place by the pair of diagonal arrows. But the only thing in the intersection of the image of $p_{1}^{*}$ and $p_{2}^{*}$ is zero; hence $\eta$ must lift to a form in $\Omega^{1}(X)$, so $\Omega^{1}(X)$ is precisely the equalizer of the vertical arrows. The cases $q>0$ follow from applying the (exact) wedge product functor. 
For general $u$, the lemma gives $\Omega^{1}(X) \hookrightarrow \Omega^{1}(Y)$. The image of $\Omega^{1}(X)$ is by definition in the intersection of all equalizers. Conversely, suppose that $\omega \in \Omega^{1}(Y)$ is in the equalizer of every pair of arrows $f, g: Z \rightrightarrows Y \rightarrow X$. Generic smoothness and the case of a smooth morphism show that the result is true at the generic point. The proposition then follows from the following lemma.

Lemma 4.3. Suppose $f: Y \rightarrow X$ is an h-covering of smooth $\mathbb{C}$-schemes with $X$ irreducible. Let $\left\{Y_{i}\right\}$ be the set of components of $Y$ which dominate $X$. Then the diagram

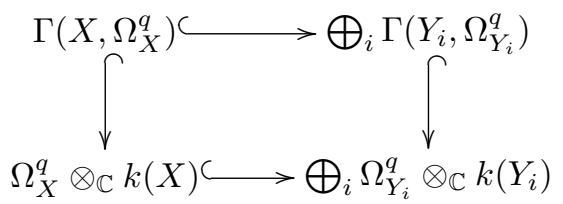

is cartesian: if a q-form $\omega$ on the generic point of $X$ lifts to a q-form on the generic point of $Y$ that extends to all of $Y$, then $\omega$ extends to all of $X$.

Proof. By Hartog's theorem ${ }^{1}$ we may safely throw out codimension $\geqslant 2$ subsets of $X$. Hence if $X^{\prime} \subset X$ is the open set where $\omega \in \Omega_{X}^{q} \otimes_{\mathbb{C}} k(X)$ is defined, then we may assume that the complement $D=X-X^{\prime}$ is a union of finitely many smooth divisors (throwing out singular and intersection sets). We may extend over one divisor at a time, so assume $D$ is a single smooth divisor.

Note it is enough to prove the lemma after replacing $Y$ with any subscheme that dominates $X$ so that $E=f^{-1}(D)$ is non-empty. Throwing out closed subsets, we may assume that $E$ is a divisor. Let $\phi: E \rightarrow D$ be $f$ restricted to $E$. Generic smoothness gives a point $y \in E$ where $\phi$ is smooth over $x=\phi(y) \in D$. We choose a complementary subspace to $m_{E, y} / m_{E, y}^{2} \subset m_{Y, y} / m_{Y, y}^{2}$, and lift generators of this subspace to equations $g_{1}, \ldots, g_{r}$ in $\mathscr{O}_{Y, y}$. We replace $Y$ with a subvariety defined by the $g_{i}$ in some neighborhood of $y \in Y$ where the $g_{i}$ are defined; thus we can assume that $\operatorname{dim} Y=\operatorname{dim} X$ and, throwing out codim $\geqslant 2$ points of $X$ and closed subsets of $Y$, we may assume that $Y$ is smooth and connected, $E$ is a smooth connected divisor, and $\phi$ is étale at $y$.

The theorem on the dimension of fibers of a morphism ([16, II ex 3.22]) gives the subset of $U \subset X$ where $f$ is quasi-finite is open. The complement $C=X-U$ is at worst dimension $\operatorname{dim} X-1$. If it is equal to $\operatorname{dim} X-1$, then its preimage is also $\operatorname{dim} X-1=\operatorname{dim} Y-1$, so applying the theorem again to components of $C$ we get a dense open set of $C$ where $f$ is quasi-finite: thus the subset of $X$ where $f$ is not quasi-finite is at least codimension 2 and we may safely throw that out, so we may assume that $f$ is quasi-finite.

By Zariski's Main Theorem ([15, 4.4.3] or [21, III.9.I]) we have a factorization $Y \subset \widetilde{X} \stackrel{\pi}{\rightarrow} X$, where $Y$ is an open immersion in the normalization $\widetilde{X}$ of $X$ in $k(Y)$. Let $E^{\prime}=\widetilde{X}-Y$. Let $W=\pi\left(E^{\prime}\right)-D$. Since by Hartog's theorem we only have to extend across the generic point of $D$, we may throw out $W$. Hence we may assume that $\pi\left(E^{\prime}\right) \cap D$ is either empty or else is all of $D$. Throwing out more points, we may assume $E$ and $E^{\prime}$ are disjoint smooth divisors. Again we only have to extend over

${ }^{1}$ or the algebraic version regarding normal varieties and codimension $\geqslant 2$ sets; see [16, II.8.19]. 
the generic point of $D$, so we may assume $X$ and $\widetilde{X}$ are affine. Let $h, h^{\prime}$ be defining equations for $E, E^{\prime}$; these exist since the stalk of $f_{*} \mathscr{O}_{Y}$ over $\mathscr{O}_{X, D}$ is a semi-local PID. We may assume $\left.h\right|_{E^{\prime}}=1=\left.h^{\prime}\right|_{E}$.

We have an $\omega \in \Omega_{X / \mathbb{C}}^{q} \otimes_{\mathbb{C}} k(X)$ so that $f^{*} \omega$ extends to an $\eta \in \Gamma\left(Y, \Omega_{Y / \mathbb{C}}^{q}\right)$. Then, for some $m$ large enough, $h^{\prime m} \eta \in \Gamma\left(\widetilde{X}, \Omega_{\tilde{X} / \mathbb{C}}^{q}\right)$. The theory of traces of $q$-forms (for example $[20,4.6 .7])$ gives us a $q$-form on $X$

$$
\operatorname{trace}\left(h^{\prime m} \eta\right)
$$

Away from $D$, we have

$$
\operatorname{trace}\left(h^{\prime m} \eta\right)=\operatorname{trace}\left(h^{\prime m}\right) \omega,
$$

so it is enough to show that trace $\left(h^{\prime m}\right)$ is invertible. Since we can throw out closed subsets not containing $D$, it is enough to show trace $\left.\left(h^{\prime m}\right)\right|_{D}$ is invertible. But this is just

$$
e_{E / D} \operatorname{trace}_{E / D}\left(\left.h^{\prime}\right|_{E}\right)^{m}=e_{E / D} \operatorname{deg}(E \rightarrow D)^{m}
$$

since $h^{\prime} \mid E=1$, where $e_{E / D}$ is the ramification.

Remark 4.4. We have a complex of sheaves $\Omega^{\bullet}$ on $\mathrm{Sm}_{h}$ and an augmentation

$$
0 \rightarrow \mathbb{C} \rightarrow \mathscr{O} \rightarrow \Omega^{1} \rightarrow \Omega^{2} \rightarrow \cdots
$$

coming from the usual inclusions and exterior differentiation. The complex $\Omega^{\bullet}$ has a natural filtration, the filtration bête.

Fix an $X \in \mathrm{Sch}$. For simplicity, we assume that $X$ is irreducible. We consider the sites $\mathrm{Sm}_{h} / X$ of smooth $\mathbb{C}$-schemes over $X, \mathrm{Sch}_{h} / X$ all $\mathbb{C}$-schemes over $X$, and $X_{\text {Zar }}$ the small site of Zariski-open subsets of $X$. The natural inclusion $\gamma: X_{\text {Zar }} \hookrightarrow \operatorname{Sch}_{h} / X$ gives $X_{\text {Zar }}$ the induced Grothendieck topology, since a family of Zariski open sets is a Zariski cover only if it is an $h$-cover. Therefore, $\gamma$ is continuous [11, ex III 3.1] and induces a geometric morphism of topoi [11, ex III 1.2.1], which we also denote by $\gamma$ :

$$
\gamma=\left(\gamma^{*}, \gamma_{*}\right): \operatorname{Sh~Sm}_{h} / X \simeq \operatorname{Sh~Sch}_{h} / X \rightarrow \operatorname{Sh} X_{\mathrm{Zar}},
$$

with the first equivalence being given by Corollary 3.7. Perhaps confusingly, for an $h$-sheaf $F$, we have $\gamma_{*} F=F \circ \gamma$. Note that

$$
\mathbb{Z} X_{h}=\gamma^{*} \mathbb{Z} X_{\text {Zar }}
$$

as both are the sheaf of free abelian groups associated to the constant presheaf with value $\mathbb{Z}$.

Remark 4.5. Since $\Omega^{q}$ is a sheaf on $\mathrm{Sm}_{h}$, for any $X \in$ Sch and any diagram

$$
X \leftarrow X_{0} \leftleftarrows X_{0} \times_{X} X_{0} \leftarrow X_{1},
$$

where $X_{0} \rightarrow X$ is an $h$-covering and $X_{0}, X_{1} \in \mathrm{Sm}, \gamma_{*} \Omega_{X}^{q}$ is determined by the exact sequence

$$
0 \rightarrow \Gamma\left(X, \gamma_{*} \Omega_{X}^{q}\right) \rightarrow \Gamma\left(X_{0}, \Omega_{X_{0} / \mathbb{C}}^{q}\right) \rightrightarrows \Gamma\left(X_{1}, \Omega_{X_{1} / \mathbb{C}}^{q}\right) .
$$

This shows that $\gamma_{*} \Omega_{X}^{q}$ is quasi-coherent. Since by $[2,17]$ we can choose proper $h$ covers, $\gamma_{*} \Omega_{X}^{q}$ is coherent. 


\subsection{Results of Du Bois}

Definition 4.6. Let $X$ be a $\mathbb{C}$-scheme. A Du Bois cover of $X$ is a smooth representable $h$-hypercover $Z_{\bullet} \rightarrow X$ with components quasi-projective and proper over $X$.

Theorem 4.7 ([5, 3.11]). Let $X$ be a $\mathbb{C}$-scheme, and $e: K_{\bullet} \rightarrow X, e^{\prime}: K_{\bullet}^{\prime} \rightarrow X$ be two $D u$ Bois covers of $X$. Let $\alpha: K_{\bullet}^{\prime} \rightarrow K_{\bullet}$ be a map over $X$. Then the induced map

$$
\operatorname{Re}_{*}\left(\Omega_{K_{\bullet} / \mathbb{C}}^{p}\right) \rightarrow \operatorname{Re}_{*}^{\prime}\left(\Omega_{K_{\bullet} / \mathbb{C}}^{p}\right)
$$

is an isomorphism in the derived category.

The morphism is constructed by applying $R e_{*}$ to

$$
\Omega_{K_{\bullet} / \mathbb{C}}^{p} \rightarrow \alpha_{*} \Omega_{K_{\bullet}^{\prime} / \mathbb{C}}^{p} \rightarrow R \alpha_{*} \Omega_{K_{\bullet}^{\prime} / \mathbb{C}}^{p}
$$

This direct image is computed in the Zariski topology; by GAGA [10] this commutes with analytification, since all components are proper over the base $X$.

Corollary 4.8 ([5, 3.17]). Under the hypotheses of Theorem 4.7, for the filtration bette on the complexes $\Omega_{K_{\bullet} / \mathbb{C}}^{\bullet}, \Omega_{K_{\bullet} / \mathbb{C}}$ the canonical map

$$
\operatorname{Re}_{*}\left(\Omega_{K_{\bullet} / \mathbb{C}}^{\bullet}\right) \rightarrow \operatorname{Re}_{*}^{\prime}\left(\Omega_{K_{\bullet} / \mathbb{C}}^{\bullet}\right)
$$

is an isomorphism in the filtered derived category.

For $X$ smooth, we can take $K_{\bullet}=X_{\bullet}$ the simplicial scheme that is $X$ in all degrees; this clearly is a smooth resolution of $X$. In this case the theorems degenerate to

Proposition 4.9. For $X$ a smooth $\mathbb{C}$-scheme and Du Bois cover $e^{\prime}: K_{\bullet}^{\prime} \rightarrow X$, we have

$$
e_{*}^{\prime} \Omega_{K_{\bullet}^{\prime} / \mathbb{C}}^{q}=\Omega_{X / \mathbb{C}}^{q}
$$

and $R^{i} e_{*}^{\prime} \Omega_{K_{\bullet}^{\prime} / \mathbb{C}}^{q}=0$ for $i>0$

Corollary 4.10 (Same hypotheses as above). Giving the complexes $\Omega_{K_{\bullet}^{\prime} / \mathbb{C}}^{\bullet}, \Omega_{X / \mathbb{C}}^{\bullet}$ the filtration bette, the canonical map

$$
\operatorname{Re}_{*}\left(\Omega_{K_{\bullet}^{\prime} / \mathbb{C}}^{\bullet} \rightarrow \Omega_{X / \mathbb{C}}^{\bullet}\right.
$$

is an isomorphism in the filtered derived category.

\subsection{Comparison of $h$ - and Zariski topology.}

We begin with a few technical lemmas. 
Lemma 4.11. Given a commutative diagram

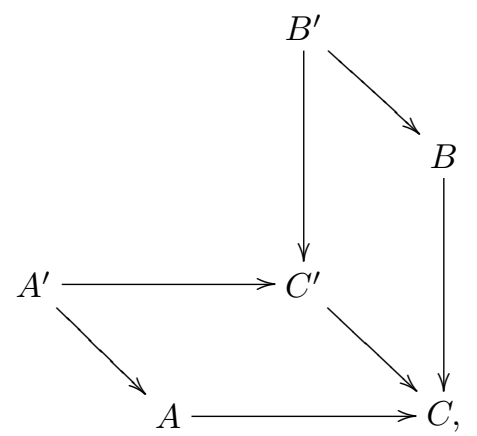

where all the diagonal arrows are Zariski covers, then $A^{\prime} \times_{C^{\prime}} B^{\prime} \rightarrow A \times_{C} B$ is a Zariski cover.

Proof. The map $A^{\prime} \rightarrow A \times_{C} C^{\prime}$ is a Zariski open cover because the composite with the projection to $A$ is an open cover, and $A \times{ }_{C} C^{\prime} \rightarrow A$ itself is a Zariski open cover. ${ }^{2}$ Likewise, $B^{\prime} \rightarrow B \times{ }_{C} C^{\prime}$ is a Zariski open cover. Thus the map

$$
A^{\prime} \times_{C^{\prime}} B^{\prime} \rightarrow\left(A \times_{C} C^{\prime}\right) \times_{C^{\prime}}\left(B \times_{C} C^{\prime}\right)=\left(A \times_{C} B\right) \times_{C} C^{\prime}
$$

is a Zariski open cover. But $\left(A \times_{C} B\right) \times_{C} C^{\prime} \rightarrow A \times_{C} B$ is a Zariski open cover by base change, hence so is $A^{\prime} \times_{C^{\prime}} B^{\prime} \rightarrow A \times_{C} B$.

Lemma 4.12. Let $X$ be a $\mathbb{C}$-scheme and $K^{\bullet} \rightarrow X$ a smooth representable h-hypercover. Then there is a diagram

$$
Z_{\bullet} \stackrel{\pi}{\leftarrow} L_{\bullet} \stackrel{\phi}{\rightarrow} K_{\bullet},
$$

where $L_{\bullet}$ is a smooth representable h-hypercover of $X, Z_{\bullet}$ is a Du Bois cover of $X$, and $\pi$ is a local acyclic fibration in the Zariski topology.

Proof. In degree zero, form the diagram of smooth $\mathbb{C}$-schemes

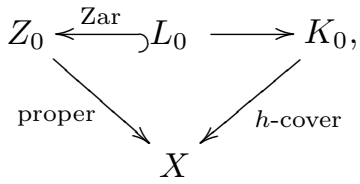

which exists by Corollary 3.15. (Here "Zar" indicates a Zariski open cover and "proper" indicates a proper surjective cover). Assume inductively that we have constructed a diagram of $n$-truncated objects

$$
Z_{\leqslant n} \stackrel{\pi_{\leqslant n}}{\leftarrow} L_{\leqslant n} \rightarrow K_{\leqslant n}
$$

where

1. All objects are smooth representable and the $K_{\leqslant n}$ is the truncation of the $K_{\bullet}$.

2. $Z_{\leqslant n}$ is an $n$-truncated Du Bois cover of $X$.

3. $L_{i} \rightarrow Z_{i}$ is a Zariski open cover for all $0 \leqslant i \leqslant n$.

${ }^{2}$ It is an étale surjective monomorphism on components. 
Note that Part 3 implies $L_{\leqslant n} \rightarrow Z_{\leqslant n}$ is a local acyclic fibration: for $k>n$ the condition

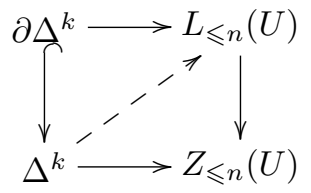

is empty, and for $k \leqslant n$ a local section $Z_{k}(V) \rightarrow L_{k}(V)$ allows us to lift.

Recall that the $i_{n *}$ are right adjoints of "taking the degree $n$ component". They are constructed by finite products and fiber products of the $L_{k}$ 's and $Z_{k}$ 's, and the morphism is component-by-component, and these are all Zariski open covers. Hence Lemma 4.11 shows that $\left(i_{n *} L_{\leqslant n}\right)_{n+1} \rightarrow\left(i_{n *} Z_{\leqslant n}\right)_{n+1}$ is a Zariski cover.

Now construct the commutative diagram

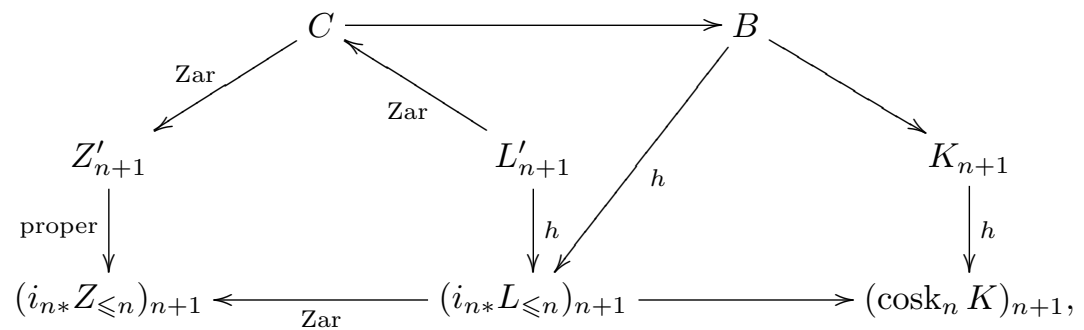

where

1. Zar indicates an arrow is a Zariski open cover, "proper" a proper surjective cover, and $h$ an $h$-cover;

2. $B$ is the fiber product $K_{n+1} \times\left(\operatorname{cosk}_{n} K\right)_{n+1}\left(i_{n *} L_{\leqslant n}\right)_{n+1}$;

3. $C \stackrel{\text { Zar }}{\longrightarrow} Z_{n+1}^{\prime} \stackrel{\text { proper }}{\longrightarrow}\left(i_{n *} Z_{\leqslant n}\right)_{n+1}$ is the factorization of the $h$-covering

$$
B \rightarrow\left(i_{n *} Z_{\leqslant n}\right)_{n+1}
$$

given by Corollary 3.15, so $Z_{n+1}^{\prime}$ is smooth representable proper over

$$
\left(i_{n *} Z_{\leqslant n}\right)_{n+1}
$$

with quasi-projective components;

4. $L_{n+1}^{\prime}$ is the fiber product $C \times_{\left(i_{n *} Z_{\leqslant n}\right)_{n+1}}\left(i_{n *} L_{\leqslant n}\right)_{n+1}$. In particular, it is an open Zariski cover of $C$, and hence smooth.

Then up to degeneracies, $L_{n+1}^{\prime}$ and $Z_{n+1}^{\prime}$ satisfy all the conditions needed: $Z_{n+1}^{\prime}$ is quasi-projective and proper and surjective over the coskeleton and $X$, and $L_{n+1}^{\prime}$ is a Zariski cover of $Z_{n+1}^{\prime}$ and completes $L_{\leqslant n}$ to a truncated $h$-hypercover. By Remark 2.14 we can fulfill degeneracy conditions by adding disjoint unions with lower degree pieces, which does not affect any of the properties we have established.

Finally, we have to show that the map $L_{n+1}^{\prime} \rightarrow K_{n+1}$ is compatible with the face maps. In other words, the direct map $L_{n+1}^{\prime} \rightarrow\left(i_{n *} L_{\leqslant n}\right)_{n+1}$ given by the vertical 
arrow factors through $B$, e.g., is equal to

$$
L_{n+1}^{\prime} \rightarrow C \rightarrow B \rightarrow\left(i_{n *} L_{\leqslant n}\right)_{n+1} .
$$

This can be checked as follows: first we simplify the notation in the diagram

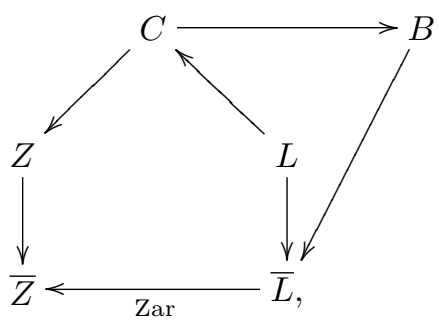

where $Z=Z_{n+1}, \bar{Z}=\left(i_{n *} Z_{\leqslant n}\right)_{n+1}$ and likewise for $L$. We write the composites $C \rightarrow B \rightarrow \bar{L}$ as $C B \bar{L}$, et cetera. Then by construction $C B \overline{L Z}=C Z \bar{Z}$, and $L C Z \bar{Z}=$ $L \overline{L Z}$. Hence $L C B \overline{L Z}=L \overline{L Z}$; but $\overline{L Z}$ is an epimorphism, so we can right-cancel, yielding $L C B \bar{L}=L \bar{L}$, which is what we wanted. Hence the map $L_{n+1}^{\prime} \rightarrow K_{n+1}$ is consistent with face maps. Compatibility with the degeneracies is automatic by the splitting construction.

The following is the main result of this section and comes from generous suggestion of Alexander Beilinson.

Theorem 4.13. Let $X$ be a $\mathbb{C}$-scheme, and $\mathscr{F} \bullet$ a bounded below complex of sheaves of abelian groups in $\mathrm{Sm}_{h} / X$ given the filtration bete. Let $Q(X)$ be the subcategory of Du Bois covers of $X$ in $R_{\mathrm{Sm}}(X)$, the category of representable smooth h-hypercovers of $X$. Then the associated homotopy category of cochain complexes $h Q(X)$ is cofiltered, and there is a filtered quasi-isomorphism of ind-objects

$$
\left.\operatorname{ind}_{Z \bullet h Q(X)} R \operatorname{Hom}_{\mathrm{Zar}}\left(\mathbb{Z} Z^{\bullet}, \mathscr{F}^{\bullet}\right)\right) \simeq R \operatorname{Hom}_{h}(\mathbb{Z} X, \mathscr{F} \bullet) .
$$

Proof. For any smooth representable $h$-hypercover $K_{\bullet} \rightarrow X$, construct

$$
Z_{\bullet} \stackrel{\pi}{\leftarrow} L_{\bullet} \stackrel{\phi}{\rightarrow} K_{\bullet}
$$

as in Lemma 4.12. This shows the $L_{\bullet}$ are cofinal in all smooth representable hypercovers, so by Corollary 3.18 we have a filtered quasi-isomorphism

$$
\underset{L^{\bullet}}{\operatorname{ind}} \operatorname{Tot} \operatorname{Hom}\left(\mathbb{Z} L^{\bullet}, \mathscr{F}^{\bullet}\right) \simeq R \operatorname{Hom}_{h}\left(\mathbb{Z} X, \mathscr{F}^{\bullet}\right)
$$

Note of course that $\operatorname{Tot} \operatorname{Hom}\left(\mathbb{Z} L^{\bullet}, \ldots\right)$ does not see the topology.

Now there is also a natural morphism

$$
\operatorname{ind}_{L^{\bullet}} \operatorname{Tot} \operatorname{Hom}\left(\mathbb{Z} L^{\bullet}, \mathscr{F}^{\bullet}\right) \rightarrow \operatorname{ind}_{Z^{\bullet}} R \operatorname{Hom}_{\mathrm{Zar}}\left(\mathbb{Z} Z^{\bullet}, \mathscr{F}^{\bullet}\right),
$$

where the $Z_{\bullet}$ run over Du Bois covers of $X$. Each $L_{\bullet}$ is a Zariski local acyclic fibration of some $Z_{\bullet}$, which gives the map. We claim in the limit that this is a filtered quasiisomorphism. This is because we can compute $R \operatorname{Hom}_{\mathrm{Zar}}\left(\mathbb{Z} Z^{\bullet}, \mathscr{F} \bullet\right)$ as the limit of Čech cohomology over Zariski local fibrations $L_{\bullet} \rightarrow Z_{\bullet}$ by Corollary 3.18, and every 
such $L_{\bullet}$ appears on the left side. The composition gives the desired filtered quasiisomorphism

$$
\left.\operatorname{ind}_{Z \bullet \in h Q(X)} R \operatorname{Hom}_{\mathrm{Zar}}\left(\mathbb{Z} Z^{\bullet}, \mathscr{F} \bullet\right)\right) \simeq R \operatorname{Hom}_{h}(\mathbb{Z} X, \mathscr{F} \bullet
$$

\subsection{Algebraic de Rham complex}

Proposition 4.14. Let $X$ be a $\mathbb{C}$-scheme. Then for any Du Bois cover $Z \bullet$ of $X$, we have a filtered quasi-isomorphism

$$
R \operatorname{Hom}_{\mathrm{Zar}}\left(\mathbb{Z} Z^{\bullet}, \Omega^{\bullet}\right) \simeq R \operatorname{Hom}_{h}\left(\mathbb{Z} X, \Omega^{\bullet}\right) .
$$

Proof. By the result of Du Bois (Corollary 4.8), every term in the ind-object of Theorem 4.13

$$
\operatorname{ind}_{Z \bullet \in h Q(X)} R \operatorname{Hom}_{\mathrm{Zar}}\left(\mathbb{Z} Z^{\bullet}, \Omega^{\bullet}\right)
$$

is isomorphic (recall $Q(X)$ is the category of Du Bois covers of $X$ ).

Definition 4.15. Let $e: Z \bullet \rightarrow X$ be a Du Bois cover of $X$, and define the algebraic de Rham complex as

$$
\underline{\Omega_{X}^{\bullet}}:=R e_{*} \Omega_{Z_{\bullet} / \mathbb{C}}^{\bullet}
$$

Recall that $\gamma_{*}: \mathrm{Sh} \mathrm{Sm}_{h} / X \rightarrow \operatorname{Sh} X_{\mathrm{Zar}}$ is the direct image of sheaves on the smooth $h$-site over $X$ to sheaves on the small Zariski site of $X$.

Theorem 4.16. The algebraic de Rham complex is quasi-isomorphic in the filtered derived category to $R \gamma_{*} \Omega^{\bullet}$ with the filtration bette.

Proof. Apply the previous proposition Zariski locally on the base $X$.

Corollary 4.17. Algebraic de Rham cohomology, with the filtration bête, is computed by the hypercohomology of $\Omega^{\bullet}$ in $\mathrm{Sm}_{h} / X$ with the filtration bete.

Proof.

$$
\mathbf{H}_{h}^{i}\left(\operatorname{Sm}_{h} / X, \Omega^{\bullet}\right)=\mathbf{H}^{i}\left(X_{\mathrm{Zar}}, R \gamma_{*} \Omega^{\bullet}\right)=\mathbf{H}^{i}\left(X_{\mathrm{Zar}}, R e_{*} \Omega_{Z \bullet}^{\bullet} \mathbb{C}\right)=H_{d R}^{i}(X) .
$$

Remark 4.18. As noted before, this filtration is typically not the Hodge filtration.

\subsection{Questions}

1. For an open $\mathbb{C}$-scheme $X$, is there a site of "log $h$-covers of $X$ " which takes the place of Deligne's construction $([3,4])$ of smooth hypercovers with boundary a normal crossing divisor?

2. Is there a model-theoretic generalization of Lemma 2.7? Is there a relative version which computes higher direct images?

3. What are the minimum hypotheses about $\Omega^{q}$ which allow the Du Bois results to go through? Is the following enough: $\mathscr{F}$ is sheaf of $\mathscr{O}$-modules on $\mathrm{Sm}_{h}$, locally free on smooth Zariski sites, with "transfers"?

4. Is there a difference between the $\mathrm{Sm}_{u c d}$ and $\mathrm{Sm}_{h}$ ? 
5. Is there a characterization of hypercovers in terms of ordinary covers if one works with the geometric realization?

6. The genesis of all of this work was an idea of Nori, on "holomorphic Whitney forms". The basic idea was to look at functionals on cycles which "vary holomorphically", in analogy with [24]; a discussion will be forthcoming in a future article. What is the relationship between this theory, "holomorphic Whitney forms," and intersection cohomology sheaves?

\section{References}

[1] B. Conrad, Cohomological descent. Available at http://math. stanford. edu/ conrad/papers/hypercover.pdf.

[2] A.J. de Jong, Smoothness, semi-stability and alterations, Inst. Hautes Études Sci. Publ. Math. (1996), no. 83, 51-93. MR1423020 (98e:14011)

[3] P. Deligne, Théorie de Hodge. II, Inst. Hautes Études Sci. Publ. Math. 40 (1971), 5-57. MR0498551 (58 \#16653a)

[4] P. Deligne, Théorie de Hodge. III, Inst. Hautes Études Sci. Publ. Math. 44 (1974), 5-77. MR0498552 (58 \#16653b)

[5] P. Du Bois, Complexe de de Rham filtré d'une variété singulière, Bull. Soc. Math. France 109 (1981), no. 1, 41-81. MR613848 (82j:14006)

[6] D. Dugger, S. Hollander, and D.C. Isaksen, Hypercovers and simplicial presheaves, Math. Proc. Cambridge Philos. Soc. 136 (2004), no. 1, 9-51. MR2034012 (2004k:18007)

[7] D. Dugger and D.C. Isaksen, Weak equivalences of simplicial presheaves, Contemp. Math., vol. 346, Amer. Math. Soc., Providence, RI, 2004, Homotopy theory: relations with algebraic geometry, group cohomology, and algebraic $K$ theory, pp. 97-113. MR2066498 (2005e:18018)

[8] S.I. Gelfand and Yu.I. Manin, Methods of homological algebra, Second Edition, Springer Monographs in Math., Springer-Verlag, New York, 2003. MR1950475 (2003m:18001)

[9] A. Grothendieck, On the de Rham cohomology of algebraic varieties, Inst. Hautes Études Sci. Publ. Math. 29 (1966), 95-103. MR0199194 (33 \#7343)

[10] A. Grothendieck (ed.), Revêtements étales et groupe fondamental (SGA 1), Documents Mathématiques (Paris), 3, Sociéte Mathématique de France, Paris, 2003. Séminaire de géométrie algébrique du Bois Marie 1960-61. Directed by A. Grothendieck, with two papers by M. Raynaud, Updated and annotated reprint of the 1971 original [Lecture Notes in Math. 224, Springer-Verlag, New York, MR0354651 (50 \#7129)]. MR2017446 (2004g:14017)

[11] A. Grothendieck (ed.), Théorie des topos et cohomologie étale des schémas. Tome 1: Théorie des topos, Springer-Verlag, New York, 1972. Séminaire de Géométrie Algébrique du Bois-Marie 1963-1964 (SGA 4), Dirigé par M. Artin, A. Grothendieck, et J.L. Verdier. Avec la collaboration de N. Bourbaki, P. Deligne et B. Saint-Donat, Lecture Notes in Math. 269. MR0354652 (50 \#713) 
[12] A. Grothendieck (ed.), Théorie des topos et cohomologie étale des schémas. Tome 2, Springer-Verlag, New York, 1972. Séminaire de Géométrie Algébrique du BoisMarie 1963-1964 (SGA 4), Dirigé par M. Artin, A. Grothendieck et J.L. Verdier. Avec la collaboration de N. Bourbaki, P. Deligne et B. Saint-Donat, Lecture Notes in Math. 270. MR0354653 (50 \#7131)

[13] A. Grothendieck (ed.), Théorie des topos et cohomologie étale des schémas. Tome 3, Springer-Verlag, New York, 1973. Séminaire de Géométrie Algébrique du BoisMarie 1963-1964 (SGA 4), Dirigé par M. Artin, A. Grothendieck et J.L. Verdier. Avec la collaboration de P. Deligne et B. Saint-Donat, Lecture Notes in Math. 305. MR0354654 (50 \#7132)

[14] A. Grothendieck, Éléments de géométrie algébrique. II. Étude globale élémentaire de quelques classes de morphismes, Inst. Hautes Études Sci. Publ. Math. (1961), no. 8, 222. MR0217084 (36 \#177b)

[15] A. Grothendieck, Éléments de géométrie algébrique. IV. Étude locale des schémas et des morphismes de schémas. III, Inst. Hautes Études Sci. Publ. Math. (1966), no. 28, 255. MR0217086 (36 \#178)

[16] R. Hartshorne, Algebraic geometry, Springer-Verlag, New York, 1977. Graduate Texts in Math. 52. MR0463157 (57 \#3116)

[17] H. Hironaka, Resolution of singularities of an algebraic variety over a field of characteristic zero. I, II, Ann. of Math. (2) 79 (1964), 109-203; ibid. (2) 79 (1964), 205-326. MR0199184 (33 \#7333)

[18] J.F. Jardine, Simplicial presheaves, J. Pure Appl. Algebra 47 (1987), no. 1, 3587. MR906403 (88j:18005)

[19] M. Kashiwara and P. Schapira, Sheaves on manifolds, Grundlehren der Mathematischen Wissenschaften, vol. 292, Springer-Verlag, New York, 1994. MR1299726 (95g:58222)

[20] J. Lipman, Residues and traces of differential forms via Hochschild homology, Contemp. Math., vol. 61, Amer. Math. Soc., Providence, RI, 1987. MR868864 (88b:14017)

[21] D. Mumford, The red book of varieties and schemes, expanded, Lecture Notes in Math., vol. 1358, Springer-Verlag, New York, 1999. MR1748380 (2001b:14001)

[22] A. Suslin and V. Voevodsky, Relative cycles and Chow sheaves, Ann. of Math. Stud., vol. 43, Princeton Univ. Press, Princeton, NJ, 2000, Cycles, transfers, and motivic homology theories, 2000, pp. 10-86. MR1764199

[23] V. Voevodsky, Homology of schemes, Selecta Math. (N.S.) 2 (1996), no. 1, 111153. MR1403354 (98c:14016)

[24] H. Whitney, Geometric integration theory, Princeton University Press, Princeton, NJ, 1957. MR0087148 (19,309c)

Ben Lee ben_lee@post.harvard.edu

530 Stockton St. Apt. 407, San Francisco, CA USA 94108 